\title{
Wireless Telemetry and Cystic Fibrosis: Just the pHacts
}

\author{
Jonathan D. Kaunitz $\cdot$ Yasutada Akiba
}

Received: 30 April 2013/Accepted: 2 May 2013/Published online: 9 June 2013

(C) Springer Science+Business Media New York (Outside the USA) 2013

Cystic fibrosis (CF) is a genetic disease affecting $~ 0.01 \%$ of the US population. Although the disease is unambiguously linked to dysfunctional mutations of the gene encoding the cystic fibrosis transmembrane regulator (CFTR), the pathobiology of its phenotype remains incompletely understood. Since one of the known functions of the CFTR is anion transport, CFTR dysfunction, with impairment of chloride and water transport, has been implicated in the genesis of the thickened mucus that characterizes CF. Since the CFTR also conducts bicarbonate ion, there has been increasing recognition of the importance of $\mathrm{pH}$ regulation at the epithelial surfaces expressing CFTR.

In the foregut, bicarbonate is secreted from the salivary, esophageal submucosal and duodenal Brunner's glands, the gastric mucosa, the duodenal epithelium, the bile ducts, and the pancreatic ductal epithelium. In CF with severe

J. D. Kaunitz · Y. Akiba

Greater Los Angeles Veterans Affairs Healthcare System, Los Angeles, CA, USA

J. D. Kaunitz · Y. Akiba

Departments of Medicine, University of California Los Angeles School of Medicine, Los Angeles, CA, USA

\section{J. D. Kaunitz}

Departments of Surgery, University of California Los Angeles School of Medicine, Los Angeles, CA, USA

J. D. Kaunitz · Y. Akiba

Brentwood Biomedical Research Institute, Los Angeles, CA 90073, USA

J. D. Kaunitz $(\square)$

West Los Angeles VA Medical Center, Bldg. 114, Suite 217,

11301 Wilshire Blvd., Los Angeles, CA 90073, USA

e-mail: jake@ucla.edu mutations, this secretion is impaired as a result of decreased CFTR bicarbonate conductance and as a consequence of the development of chronic liver disease and fibrocystic destruction of pancreatic parenchyma. Thus, CF subjects harboring severe mutations have markedly impaired foregut bicarbonate secretion in association with rapidly progressive, morbid disease. Moreover, since gastric acid secretion is unimpaired, post-pyloric luminal $\mathrm{pH}$ is decreased, with resultant fat malabsorption and steatorrhea due to reduced pancreatic enzyme secretion from acinar destruction, luminal pancreatic enzyme denaturation, and impaired micellar solubilization, among other factors. Fat malabsorption with its consequent malnutrition is a major contributor to CF-associated morbidity.

To improve fat absorption, pancreatic enzyme replacement has been advocated in CF patients with steatorrhea. Since pancreatic enzymes denature at $\mathrm{pH} \mathrm{4}$, they are enterically coated to protect them as they transit through the acidic stomach, the enteric coating designed to dissolve in the more neutral $\mathrm{pH}$ of the duodenum. In CF subjects, however, impaired bicarbonate secretion lowers duodenal $\mathrm{pH}$ and delay dissolution of the coating. This, in turn, delays the release of pancreatic enzymes, reducing their availability further down the intestine, impairing their digestive efficiency.

From the foregoing, the clinical importance of small intestinal $\mathrm{pH}$ regulation in $\mathrm{CF}$ subjects is obvious. Yet, few measurements have been made largely due to technologic limitations. Nevertheless, even as early as 1959 , measurements have been obtained using the "Heidelberg" radiotelemetric $\mathrm{pH}$-sensing capsule $[1,2]$. The Heidelberg capsule consisted of an antimony/silver $\mathrm{pH}$ electrode with battery-coupled radio transmitter whose signals were detected by a receiver worn on the subject's chest. Fast forward to the present time: in the paper by Gelfond et al. 
in this issue of DDS [3], the authors report a study in which a "wireless capsule" was used to measure small intestinal luminal pH in CF patients. Given the "wireless" nature of the capsule, it further allowed them to be studied more comfortably and for intestinal $\mathrm{pH}$ to be measured serially, real-time, and so under varied conditions. The study was conducted in ten CF subjects and ten healthy controls and showed, not surprisingly, that post-pyloric (duodenal) $\mathrm{pH}$ was significantly lower in CF patients than in the controls. Indeed, small intestinal $\mathrm{pH}$ in $\mathrm{CF}$ only approximated that of controls after traveling for down the intestine for $35 \mathrm{~min}$, which corresponds to an area within the mid-jejunum. Although the mid-jejunum may not be a precise location for normalization of intestinal $\mathrm{pH}$ in the $\mathrm{CF}$ subjects-this due to a contribution by slower intestinal transit-the extended negative impact of low luminal $\mathrm{pH}$ on digestive enzyme function is fully evident.

In effect, the paper by Gelfond et al. offers new and important data on intestinal $\mathrm{pH}$ in CF subjects. Moreover, it helps confirm the findings of Youngberg et al. published in the journal in 1987. At that time, Youngberg et al. [4] showed delayed attainment of $\mathrm{pH} 5.5$ or $\mathrm{pH} 6.0$ in $\mathrm{CF}$ subjects compared with healthy controls. Also, since this study was performed prior to the availability of proton pump inhibitors (PPIs), the qualitative similarity of the data to that of Gelfond et al. supports the present studies assessment that PPI discontinuation in the CF subjects did not materially affect the data. The paper by Gelfond et al. also provides detailed documentation of duodenal $\mathrm{pH}$ in genotyped $\mathrm{CF}$ subjects, confirming and extending the observations in previous studies relating to the " $\mathrm{CF}$ Paradox"-the paradox being the clinical observation that despite impaired bicarbonate secretion associated with lower intraluminal intestinal $\mathrm{pH}, \mathrm{CF}$ subjects did not have a higher prevalence of duodenal ulcer disease $[5,6]$.

The wireless capsule (SmartPill) used by Gelfond et al., was initially developed as a tool for studying intestinal motility through the measurement of intestinal transit time-but as shown it has the additional advantage of being able to serially measure intraluminal $\mathrm{pH}$ in real-time. This latter feature enables the creation of a high-resolution axial luminal $\mathrm{pH}$ profile for the entire gastrointestinal tract, increasing its value as a research tool for the study of other clinical conditions that may impact intestinal $\mathrm{pH}$. For example, this technology would be useful in studying patients with bacterial overgrowth, those with mutations of acid or base transporters such as the gene encoding SLC26A3 (the anion exchanger affected in congenital chloride diarrhea), and those with pancreatic exocrine insufficiency or acid hypersecretory conditions, like Zollinger-Ellison syndrome (ZES). Furthermore, a high-resolution luminal $\mathrm{pH}$ profile may have utility in the design of enteric coatings for medications and in quantifying the efficacy of anti-secretory and pro-secretory drugs for raising and lowering small intestinal $\mathrm{pH}$.

Above all, the study by Gelfond et al. illustrates the power of modern technology to address clinical questions in novel and insightful ways and in areas of the GI tract that have heretofore been difficult to reach. With further refinements of capsule technology, such as adding cameras, chemical sensors, and fluid-collecting functions, we have every reason to suspect that there is a great deal more to come.

\section{References}

1. Nöller HG. Endoradiosound technic and its significance for internal medicine. Verh Dtsch Ges Inn Med. 1959;65:727-730.

2. Stack BH. Use of the Heidelberg $\mathrm{pH}$ capsule in the routine assessment of gastric acid secretion. Gut. 1969;10:245-246.

3. Gelfond D, Ma C, Semler J, Borowitz D. Intestinal pH and gastrointestinal transit profiles in cystic fibrosis patients measured by wireless motility capsule. Dig Dis Sci. (Epub ahead of print). doi:10.1007/s10620-012-2209-1.

4. Youngberg CA, Berardi RR, Howatt WF, et al. Comparison of gastrointestinal $\mathrm{pH}$ in cystic fibrosis and healthy subjects. Dig Dis Sci. 1987;32:472-480.

5. Akiba Y, Furukawa O, Guth $\mathrm{PH}$, et al. Cellular bicarbonate protects rat duodenal mucosa from acid-induced injury. J Clin Invest. 2001;108:1807-1816.

6. Kaunitz JD, Akiba Y. Duodenal intracellular bicarbonate and the “CF paradox". JOP. 2001;2:268-273. 